\title{
A Formal Total Synthesis of Deoxynojirimycin from D-Glucitol
}

\author{
Raquel M. Braga* and Lucilia Kato \\ Instituto de Química, Universidade Estadual de Campinas, CP 6154, 13084-971 Campinas - SP, Brazil
}

\begin{abstract}
Descrevemos a síntese formal da desoxinojirimicina usando D-glucitol como material de partida economicamente atraente. Através de uma seqüência de reações de proteção e desproteção seletivas e reações de substituição nucleofílica obtivemos diversos intermediários isopropilidênicos. A formação de um epóxido intermediário e sua subseqüente abertura, através de uma reação nucleofílica intramolecular, levou à obtenção do heterociclo 1,5-didesoxi-1,5-diamino-2,3-O-isopropilideno-6silila-D-glucitol, que é um precursor da desoxinojirimicina (DNJ).
\end{abstract}

This report deals with the formal synthesis of deoxynojirimycin using D-glucitol as an inexpensive starting material. Through a sequence of several selective protection/deprotection and nucleophilic substitution reactions, many isopropylidene derivatives were obtained. Formation of an epoxide intermediate and its subsequent ring opening, via intramolecular nucleophilic substitution, leads to the synthesis of heterocyclic 1,5-dideoxy-1,5-diamine-2,3-O-isopropylidene-6-silyl-D-glucitol, which is a precursor of deoxynojirimycin (DNJ).

Keywords: D-glucitol, glycosidase inhibitors, isopropylidene derivatives, deoxynojirimycin

\section{Introduction}

Imino sugars, ${ }^{1}$ sometimes called azasugars, are compounds that have been shown to selectively inhibit oligosaccharide processing enzymes (i.e., glycosidases or glycotransferases). ${ }^{2-5}$ Because of the potential chemotherapeutic applications of such compounds, there is a continuing interest on the synthesis of imino sugars. Deoxynojirimycin (DNJ, 1,5-dideoxy-1,5-imino-Dglucitol) is an inhibitor for several glycosidases, and, additionally, it shows antidiabetic and antiviral activities, including anti-HIV activity. ${ }^{6-8}$ Its importance is disclosed by the large number of publications devoted to its preparation.

After Paulsen et al. ${ }^{9}$ have obtained 1- deoxynojirimycin and disclosed its potent glucosidase inhibitor effects, it has been synthesized by a variety of methods. Kinast et al. ${ }^{10}$ obtained 1 - deoxynojirimycin in four steps from D-glucose through an enzymatic oxidation. Behling et al. ${ }^{11}$ described a synthesis of DNJ (1) from L-sorbose that requires the isolation of only two intermediates and the use of only one protection/deprotection protocol. Setoi et al. ${ }^{12-14}$ described a synthesis of DNJ (1) from D-mannose by a multi-step approach with protecting/deprotecting

* e-mail: raquel@iqm.unicamp.br group manipulation. To date several syntheses of DNJ (1) and analogues have been described. ${ }^{7,15-18}$

This report deals with the formal total synthesis of DNJ (1) using D-glucitol, an inexpensive starting material, through a sequence of several selective protection/ deprotection and nucleophilic substitution reactions. ${ }^{19}$

\section{Results and Discussion}

Treatment of D-glucitol with THF, 2,2-dimethoxypropane (DMP) and $p$-toluenesulfonic acid (TsOH) resulted in diacetonides 4 (3\%), 3 (46\%) and triacetonide 2 (16\%), as shown in Scheme 1.

The 4-O-acetyl-1-azido-1-deoxy-2,3:5,6-di- $O$ isopropylidene-D-glucitol (7) was obtained from 3 in $37 \%$ overall yield by the sequence of sulfonylation, acetylation and azidation $(3 \rightarrow 5 \mathrm{a} \rightarrow 6 \rightarrow 7)$. Selective cleavage of the terminal acetonide group was accomplished using $p$-toluenesulfonic acid in methanol at $0{ }^{\circ} \mathrm{C}$ for $5 \mathrm{~h}$. The resulting mixture of acetonides $\mathbf{7}(51 \%)$ and $\mathbf{8 a}(34 \%)$ was purified by flash column chromatography. Under the applied conditions, acyl group migration from C-4 to C-6 was observed. Acyl group migration in non-acidic aqueous media or in dichloromethane containing triethylamine was also observed by Kilonda et al. ${ }^{6}$ When this reaction is carried out using pyridinium 4-toluenesulfonate as catalyst, 
acyl group migration is not observed. This migration protects the primary group at C-6 from tosylation during the next step. The azide epoxide $\mathbf{1 0}$ was obtained after sulfonation of 8a and treatment with $\mathrm{K}_{2} \mathrm{CO}_{3}$, followed by basic treatment to promote deacetylation. The trans stereochemistry of the resulting epoxide $\mathbf{1 0}$ is confirmed by the coupling constant $(\mathrm{J} 2.2 \mathrm{~Hz})$ between $\mathrm{H} 4$ and $\mathrm{H} 5 .{ }^{20,21}$ The intermediate $\mathbf{1 1}$ is obtained by protection with TBDMSCl. Treatment of the epoxide azide 11 with $\mathrm{NaBH}_{4}$ in boiling methanol gave $\mathbf{1 3}$ a protected form of DNJ (1). The intermediate epoxide amine $\mathbf{1 2}$ could not be isolated
(Scheme 1) and this reaction afforded a single piperidine heterocycle. In summary, $\mathbf{1 3}$ was obtained in 9 steps and $0.4 \%$ overall yield from D-glucitol.

Setoi et al. ${ }^{12-14}$ have obtained DNJ (1) by treatment of 13 a protected form of DNJ (1) with aqueous TFA for $12 \mathrm{~h}$, which has been prepared from D-mannose in 14 steps and $0.4 \%$ overall yield.

${ }^{1} \mathrm{H}$ NMR, ${ }^{13} \mathrm{C}$ NMR, 2D proton-proton homocorrelated and $\mathrm{C}-\mathrm{H}$ heterocorrelated spectra and comparation with literature data confirmed the structure of intermediates. ${ }^{21}$

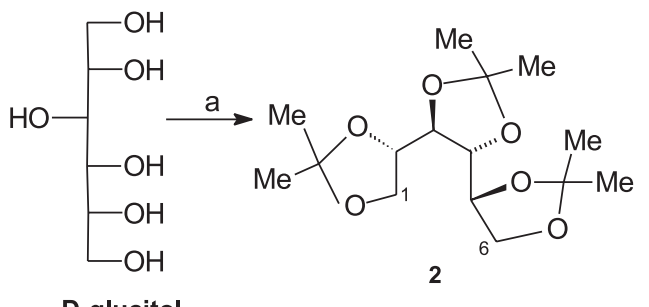

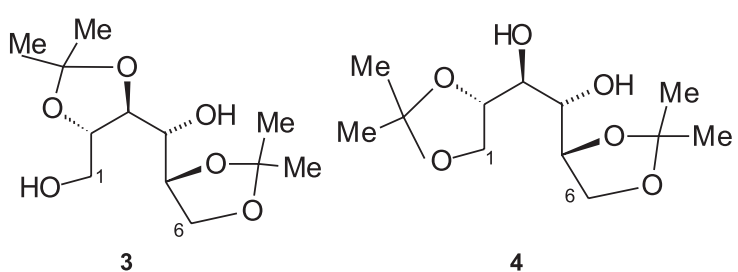

D-glucitol<smiles>CC1OC(C)(C)OC1[C@@H](O)[C@@H]1OC(C)(C)OC1CO</smiles><smiles>[R]C[C@H]1OC(C)(C)O[C@H]1[C@H]([R])[C@H]1[CH]OC(C)(C)O1</smiles><smiles>[R]C[C@H]1OC(C)(C)O[C@H]1[C@H]([R])[C@H]1COC(C)(C)O1</smiles>

5a $\mathrm{R}^{1}=\mathrm{OTs} ; \mathrm{R}^{2}=\mathrm{OH}$ 5b $R^{1}=R^{2}=O T s$

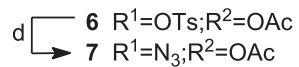<smiles>[R]CC([R])[C@H]([R])[C@H]1OC(C)(C)O[C@@H]1CN</smiles><smiles>[R]CC([R])C([R])C1OC(C)(C)OC1CN</smiles>

9a $R^{1}=O H ; R^{2}=O T s ; R^{3}=O A c ;$ 8a $R^{1}=R^{2}=O H ; R^{3}=O A c ;$
8b $R^{1}=O A c ; R^{2}=R^{3}=O T s ;$ 9b $R^{1}=R^{2}=O T s ; R^{3}=O A c$;<smiles>[R]CC1OC1C1OC(C)(C)OC1CN</smiles>
$\mathrm{R}^{1}$<smiles>CCCOCC1OC1C1OC(C)(C)O[C@@H]1CN</smiles><smiles>CCC(C)C</smiles><smiles>CC(C)OCC1NC[C@@H]2OC(C)(C)O[C@H]2[C@H]1O</smiles><smiles></smiles>

a) DMP, TsOH, THF;b) $\mathrm{TsCl}, \mathrm{Et}_{3} \mathrm{~N}$; c) $\mathrm{Ac}_{2} \mathrm{O}, \mathrm{Et}_{3} \mathrm{~N}$; d) $\mathrm{NaN}_{3}$, DMF; e) $\mathrm{MeOH}, \mathrm{TsOH}$; f) $\mathrm{K}_{2} \mathrm{CO}_{3}, \mathrm{MeOH}$; g) TBDMSCl, imidazol, DMF; h) $\mathrm{NaBH}_{4}$, EtOH, reflux 


\section{Experimental}

\section{General procedures}

Thin-layer chromatography (TLC) was carried out on plates precoated with silica gel $60 \mathrm{~F}_{254}$ (E. Merck). Detection was by UV at $254 \mathrm{~nm}$, followed by charring with a solution of ammoniun molybdate or Dragendorff reagent. ${ }^{22}$ Column chromatography was carried out on silica gel (230-400 mesh) and, unless otherwise stated, mixtures of heptane-EtOAc or $\mathrm{CH}_{2} \mathrm{Cl}_{2}-\mathrm{MeOH}$ were used as eluent. All reactions were conducted under an argon atmosphere. Anhydrous $\mathrm{Na}_{2} \mathrm{SO}_{4}$ was used to dry organic solution and the removal of the solvent was done under vacuum with a rotary evaporator. Solvents were dried and purified using standard methods. ${ }^{23}$ IR spectra were recorded with a Bomen FTIR spectrometer using $\mathrm{KBr}$ pellets or $\mathrm{NaCl}$ cells. Elemental analyses were performed on a model $2400 \mathrm{CHN}$-Perkin Elmer instrument. Nuclear magnetic resonance spectra were obtained using Varian $300 \mathrm{MHz}$ or Bruker AC-300 MHz spectrometers in $\mathrm{CDCl}_{3}$ solutions with $\left(\mathrm{CH}_{3}\right)_{4} \mathrm{Si}$ as internal reference $(300 \mathrm{MHz}$ for ${ }^{1} \mathrm{H}$ and $75 \mathrm{MHz}$ for ${ }^{13} \mathrm{C}$ ). The assignments were confirmed by proton-proton homocorrelated and carbon-proton heterocorrelated spectra. HREIMS (70 eV, direct probe) experiment was performed using a VG Auto Spec-Fisions Instrument using electron ionisation at $70 \mathrm{eV}$ (linked scan from $8 \mathrm{keV}$ collisions with helium). Optical rotations were measured with a Jasco model J-720 digital polarimeter.

1,2:5,6-di-O-isopropylidene-D-glucitol (4), 2,3:5,6-di-Oisopropylidene-D-glucitol (3) and 1,2:3,4:5,6-tri-Oisopropylidene-D-glucitol (2)

To a mixture containing $3 \mathrm{~cm}^{3}$ of THF and $1.03 \mathrm{~g} \mathrm{(5.7}$ mmol) of D-glucitol, $1.5 \mathrm{~cm}^{3}$ of DMP and TsOH were added. The mixture was stirred at $0{ }^{\circ} \mathrm{C}$ for $1 \mathrm{~h}$ and left to stand at room temperature for $12 \mathrm{~h}$. The mixture was neutralized with aqueous $\mathrm{NH}_{3}$ and evaporated. The residue was dissolved in $\mathrm{CH}_{2} \mathrm{Cl}_{2}$ and the solution was washed with saturated aqueous $\mathrm{NaCl}$ and dried over anhydrous $\mathrm{Na}_{2} \mathrm{SO}_{4}$. Purification by column chromatography gave three derivatives: 4 (0.04 g, 3\%); $3(0.667 \mathrm{~g}, 46 \%)$ and $2(0.271 \mathrm{~g}$, $16 \%)$

2. Yellowish amorphous solid; $[\alpha]_{\mathrm{D}}^{25}=+7.61^{\circ}\left(\mathrm{CHCl}_{3}\right)$; (lit. $\left.{ }^{24,25}+7.5^{\circ}\right), v_{\text {max. }} / \mathrm{cm}^{-1}: 2986,1370,1073$ (film); ${ }^{1} \mathrm{H} \mathrm{NMR}$ $\left(\mathrm{CDCl}_{3}\right) \delta 1.34\left(\mathrm{~s}, 3 \mathrm{H}, \mathrm{CH}_{3}\right), 1.38\left(\mathrm{~s}, 3 \mathrm{H}, \mathrm{CH}_{3}\right), 1.39(\mathrm{~s}, 3 \mathrm{H}$, $\left.\mathrm{CH}_{3}\right), 1.42$ (s , 3H, $\left.\mathrm{CH}_{3}\right), 1.43\left(\mathrm{~s}, 3 \mathrm{H}, \mathrm{CH}_{3}\right), 1.44(\mathrm{~s}, 3 \mathrm{H}$, $\mathrm{CH}_{3}$ ), 3.85 (dd, $J 8.0$ and $\left.7.3 \mathrm{~Hz}, 1 \mathrm{H}, \mathrm{H}-1\right), 3.97$ (dd, $J 8.0$ and $4.7 \mathrm{~Hz}, 1 \mathrm{H}, \mathrm{H}-1$ '), 4.04 (dd, $J 8.3$ and $6.7 \mathrm{~Hz}, 1 \mathrm{H}, \mathrm{H}-6)$, 4.00-4.20 (m, 4H, H-2, H-3, H-4 and H-5), 4.15 (dd, J 8.3 and $J 2.3 \mathrm{~Hz}, 1 \mathrm{H}, \mathrm{H}-6) .{ }^{13} \mathrm{C} \mathrm{NMR}\left(\mathrm{CDCl}_{3}\right) \delta: 25.3,26.6$, 25.7, 26.3, 26.9, 27.2, 66.1, 67.9, 76.8, 77.3, 78.1, 80.5, 109.5, 109.8, 109.8. Elem. Anal: Calc. for $\mathrm{C}_{15} \mathrm{H}_{26} \mathrm{O}_{6}$ : C, 59.60; H, 8.61; Found: C, 60.08; H, 8.70.

4. Colorless needles; mp $90{ }^{\circ} \mathrm{C},[\alpha]_{\mathrm{D}}{ }^{25}=+10.74^{\circ}$ $\left(\mathrm{CHCl}_{3}\right) ;{ }^{1} \mathrm{H} \mathrm{NMR}\left(\mathrm{CDCl}_{3}\right) \delta 1.35\left(\mathrm{~s}, 3 \mathrm{H}, \mathrm{CH}_{3}\right), 1.38(\mathrm{~s}, 3 \mathrm{H}$, $\left.\mathrm{CH}_{3}\right), 1.41$ (s, 3H, $\left.\mathrm{CH}_{3}\right), 1.45\left(\mathrm{~s}, 3 \mathrm{H}, \mathrm{CH}_{3}\right), 2.65$ (br, s, $1 \mathrm{H}$, $\mathrm{OH}$ ), 2.83 (br ,s, $1 \mathrm{H}, \mathrm{OH}), 3.52$ (s, 1H, H-3), 3.75 (dt, J 4.7 and $1.4 \mathrm{~Hz}, 1 \mathrm{H}, \mathrm{H}-4), 3.91$ (dd, $J 8.4$ and $7.0 \mathrm{~Hz}, 1 \mathrm{H}, \mathrm{H}-6)$, 4.02 (dd, J 7.5 and $4.7 \mathrm{~Hz}, 1 \mathrm{H}, \mathrm{H}-1$ '), 4.09 (dd, $J 8.4$ and 6.6 Hz, 1H, H-6.), 4.10-4.20 (m, 2H, H-1, H-2), 4.28 (dt, J 7.0, 6.6 and $4.2 \mathrm{~Hz}, 1 \mathrm{H}, \mathrm{H}-5) ;{ }^{1} \mathrm{H} \mathrm{NMR}\left(\mathrm{CDCl}_{3} / \mathrm{TMS} / \mathrm{D}_{2} \mathrm{O}\right) \delta$ 1.35 (s, 3H, $\left.\mathrm{CH}_{3}\right), 1.38$ (s, 3H, $\left.\mathrm{CH}_{3}\right), 1.41\left(\mathrm{~s}, 3 \mathrm{H}, \mathrm{CH}_{3}\right), 1.45$ (s, $3 \mathrm{H}, \mathrm{CH}_{3}$ ), 3.51 (dd, J 7.5 and $\left.1.4 \mathrm{~Hz}, 1 \mathrm{H}, \mathrm{H}-3\right), 3.75$ (dd, $J 4.7$ and $1.4 \mathrm{~Hz}, 1 \mathrm{H}, \mathrm{H}-4), 3.90(\mathrm{dd}, J 8.4$ and $7.0 \mathrm{~Hz}, 1 \mathrm{H}$, H-6), 4.02 (dd, J 7.5 and 4.7Hz, 1H, H-1'), 4.08 (dd, 8.4 and $\left.6.6 \mathrm{~Hz}, 1 \mathrm{H}, \mathrm{H}-6^{\circ}\right), 4.15$ (m, 2H, H-1, H-2), 4.28 (dt, J 7.0, 6.6 and $4.7 \mathrm{~Hz}, 1 \mathrm{H}, \mathrm{H}-5) ;{ }^{13} \mathrm{C} \mathrm{NMR}\left(\mathrm{CDCl}_{3}\right) \delta: 25.3,25.4,26.5$, 26.9, 66.1, 67.2, 69.4, 73.9, 75.3, 78.1, 109.5, 110.0.; Anal. Calc. for C, 54.96; H, 08.83; Found: C, 54.93; H, 08.46.

3. Colorless oil; $[\alpha]_{\mathrm{D}}{ }^{25}=-12.43^{\circ}\left(\mathrm{CHCl}_{3}\right) ;\left(\right.$ lit. ${ }^{24,25}$ $\left.-15.15^{\circ}\right) .{ }^{1} \mathrm{H} \mathrm{NMR}\left(\mathrm{CDCl}_{3}\right): \delta 1.35\left(\mathrm{~s}, 3 \mathrm{H}, \mathrm{CH}_{3}\right), 1.41(\mathrm{~s}, 3 \mathrm{H}$, $\left.\mathrm{CH}_{3}\right), 1.43\left(\mathrm{~s}, 6 \mathrm{H}, 2 \mathrm{CH}_{3}\right), 2.60(\mathrm{~m}, 2 \mathrm{H}, 2 \mathrm{OH}), 3.50(\mathrm{dt}, J 2.5$ and $6.6 \mathrm{~Hz}, 1 \mathrm{H}, \mathrm{H}-4), 3.71$ (m, 1H, H-1), 3.81 (m, 1H, H-1'), 4.00-4.20 (m, 5H, H-2, H-3, H-5, H-6 and H-6 '). ${ }^{1} \mathrm{H}$ NMR $\left(\mathrm{CDCl}_{3} / / \mathrm{D}_{2} \mathrm{O}\right) \delta: 1.35\left(\mathrm{~s}, 3 \mathrm{H}, \mathrm{CH}_{3}\right), 1.41\left(\mathrm{~s}, 3 \mathrm{H}, \mathrm{CH}_{3}\right), 1.43$ $\left(\mathrm{s}, 6 \mathrm{H}, 2 \mathrm{CH}_{3}\right), 3.50$ (dd, J 2.5 and $\left.6.6 \mathrm{~Hz}, 1 \mathrm{H}, \mathrm{H}-4\right), 3.60$ (dd, $J 4.2$ and $12.0 \mathrm{~Hz}, 1 \mathrm{H}, \mathrm{H}-1), 3.81$ (dd, $J 12.0$ and $3.6 \mathrm{~Hz}$, $1 \mathrm{H}, \mathrm{H}-1$ ') , 4.00-4.20 (5H, m, H-2, H-3, H-5, H-6, H-6'). ${ }^{13} \mathrm{C} \mathrm{NMR}\left(\mathrm{CDCl}_{3}\right) \delta: 25.1,26.6,26.8,27.1,61.9,66.9$, $70.7,76.3,77.1,77.4,109.4,109.5$.

2,3:5,6-di-O-isopropylidene-1-p-toluenesulfonyl-Dglucitol (5a) and 2,3:5,6-di-O-isopropylidene-1,4-di-ptoluenesulfonyl-D-glucitol (5b)

Method A. To a solution of diacetonide 3 (1.45 g, 5.5 $\mathrm{mmol})$ in $\mathrm{Et}_{3} \mathrm{~N}\left(5.0 \mathrm{~cm}^{3}\right)$ cooled externally by an ice bath $\mathrm{TsCl}$ was added (1.43 g) under argon. The mixture was stirred at $0{ }^{\circ} \mathrm{C}$ for $6 \mathrm{~h}$ and left to stand at room temperature for $14 \mathrm{~h}$. The mixture was dissolved in $\mathrm{CH}_{2} \mathrm{Cl}_{2}$ and the solution was washed with saturated aqueous $\mathrm{NaCl}$, dried over anhydrous $\mathrm{Na}_{2} \mathrm{SO}_{4}$ and evaporated. The residue was purified by column chromatography (6:4 heptane-EtOAc) to give 5a $(1.03 \mathrm{~g}, 45 \%)$, a monotosyl derivative and the ditosyl 5b (1.09 g, 35\%).

Method $B$. In a procedure similar to the one described above, a mixture containing $3(0.60 \mathrm{~g}, 2.29 \mathrm{mmol}), \mathrm{CH}_{2} \mathrm{Cl}_{2}$ $\left(3.0 \mathrm{~cm}^{3}\right)$, and $\mathrm{Et}_{3} \mathrm{~N}\left(0.3 \mathrm{~cm}^{3}\right)$ was added to $\mathrm{TsCl}(0.43 \mathrm{~g})$, under an inert atmosphere while being cooled externally by an ice bath. The reaction was then kept in the refrigerator 
for $14 \mathrm{~h}$. The mixture was dissolved in $\mathrm{CH}_{2} \mathrm{Cl}_{2}$ and the resulting solution was washed with saturated aqueous $\mathrm{NaCl}$, dried $\left(\mathrm{Na}_{2} \mathrm{SO}_{4}\right)$ and evaporated. The residue was purified by column chromatography (6:4 heptane-EtOAc) to give 5a $(0.60 \mathrm{~g}, 63 \%), \mathbf{5 b}(0.07 \mathrm{~g}, 2 \%)$ and $\mathbf{3}(0.15 \mathrm{~g}$, $10 \%)$.

5a. Colourless oil; $v_{\text {max }} / \mathrm{cm}^{-1}: 3512,1371,1170,1070$, 737 (film); ${ }^{1} \mathrm{H} \mathrm{NMR}\left(\mathrm{CDCl}_{3}\right) \delta: 1.39$ (s, 6H, $\mathrm{CH}_{3}$ ), 1.33 (s, $\left.3 \mathrm{H}, \mathrm{CH}_{3}\right), 1.35\left(\mathrm{~s}, 3 \mathrm{H}, \mathrm{CH}_{3}\right), 2.43\left(\mathrm{~s}, 3 \mathrm{H}, \mathrm{CH}_{3}\right), 2.28$ (br, d, $1 \mathrm{H}, \mathrm{OH}), 3.45$ (t, J3.0 and 7.6 Hz, 1H, H-4); 3.9-4.3 (m, 7H, H-1, H-1', H-2, H-3, H-5, H-6 and H-6' ), 7.38 (d, J 8.4 Hz, $2 \mathrm{H}) ; 7.81(\mathrm{~d}, J 8.4 \mathrm{~Hz}, 2 \mathrm{H}) ;{ }^{13} \mathrm{C} \mathrm{NMR}\left(\mathrm{CDCl}_{3}\right) \delta: 21.7,25.3$, 26.8, 26.9, 27.0, 67.2, 69.1, 71.3, 74.7, 76.3, 77.9, 109.8, 110.4, 128.3, 130.1, 132.1, 145.3.

$5 \boldsymbol{b}$. White amorphous solid; $[\alpha]_{\mathrm{D}}^{25}=+7.9^{\circ}$ found $\left(\mathrm{CHCl}_{3}\right)$; (lit. $\left.{ }^{24,25}+6.8^{\circ}\right) .{ }^{1} \mathrm{H} \mathrm{NMR}\left(\mathrm{CDCl}_{3}\right) \delta 1.30(\mathrm{~s}, 3 \mathrm{H}$, $\left.\mathrm{CH}_{3}\right), 1.35$ (s, 6H, $\left.\mathrm{CH}_{3}\right), 1.39$ (s, 3H, $\left.\mathrm{CH}_{3}\right), 2.45$ (s, 3H, $\mathrm{CH}_{3}$ ), 2.46 (s, 3H, $\left.\mathrm{CH}_{3}\right), 3.81$ (t, J 7.5 Hz, 1H, H-6), 3.95 (dd, J 3.8 and 7.5Hz, $2 \mathrm{H}, \mathrm{H}-4$ and H-6' ), 4.06-4.11 (m, 3H, H-1', H-3 and $\mathrm{H}-5), 4.25$ (dd, $J 11.4$ and $2.3 \mathrm{~Hz}, 1 \mathrm{H}, \mathrm{H}-1), 4.65-4.72$ (m, 1H, H-2), $7.72(\mathrm{~d}, 4 \mathrm{H}), 7.35(\mathrm{~m}, 4 \mathrm{H}) .{ }^{13} \mathrm{C} \mathrm{NMR}\left(\mathrm{CDCl}_{3}\right)$ $\delta: 21.6,21.7,25.6,26.0,26.8,27.0,65.7,67.8,74.1,74.9$, 78.7, 78.8, 109.7, 110.8, 127.9, 128.0, 129.8, 129.9, 132.1, $133.0,145.1,145.5$.

\section{4-Acetyl-2,3:5,6-di-O-isopropylidene-1-p-toluenesulfonyl- D-glucitol (6)}

To a mixture containing monotosyl derivative 5a (1.60 $\mathrm{g}, 3.85 \mathrm{mmol}$ ) and $10.0 \mathrm{~cm}^{3}$ of $\mathrm{Et}_{3} \mathrm{~N}, 1.0 \mathrm{~cm}^{3}$ of acetic anhydride was added. The mixture was stirred at room temperature for $24 \mathrm{~h}$. After that, it was neutralised with aqueous $\mathrm{NH}_{3}$ and evaporated. The residue was dissolved in $\mathrm{CH}_{2} \mathrm{Cl}_{2}$ and the solution was washed with saturated aqueous $\mathrm{NaCl}$ and dried over $\mathrm{Na}_{2} \mathrm{SO}_{4}$. The $\mathrm{Et}_{3} \mathrm{~N}$ was evaporated in a rotary evaporator using $\mathrm{MeOH}$ for the azeotropic system. Purification by a flash silica gel chromatographic column $(7: 3$ heptane-EtOAc) gave 6 (1.34 g, 76\%). $v_{\text {max. }} / \mathrm{cm}^{-1}: 2988 ; 1745 ; 1371 ; 1226 ; 1117$ (film).

4-O-acetyl-1-azido-1-deoxy-2,3:5,6 di-O-isopropylideneD-glucitol (7)

To a mixture containing $4.21 \mathrm{~g}(9.2 \mathrm{mmol})$ of acetyltosyl 6, $10.0 \mathrm{~cm}^{3}$ DMF and $2.0 \mathrm{~cm}^{3} \mathrm{H}_{2} \mathrm{O}, 0.830 \mathrm{~g} \mathrm{NaN}_{3}$ (12.7 mmol) was added. The mixture was heated under reflux for $12 \mathrm{~h}$. The solution was added to an ice-water mixture. The precipitate formed was filtered and washed with $\mathrm{CH}_{2} \mathrm{Cl}_{2}$. Purification by a flash silica gel chromatographic column (7:3 heptane-EtOAc) gave 7
(2.68 $\mathrm{g}, 86 \%)$ as an amorphous compound, white solid. $v_{\text {max }} / \mathrm{cm}^{-1}: 2988 ; 2104,1740,1372,1225,1070$ (film). ${ }^{1} \mathrm{H}$ $\operatorname{NMR}\left(\mathrm{CDCl}_{3}\right) \delta: 1.34\left(\mathrm{~s}, 3 \mathrm{H}, \mathrm{CH}_{3}\right), 1.38\left(\mathrm{~s}, 6 \mathrm{H}, \mathrm{CH}_{3}\right), 1.44$ (s, 3H, $\left.\mathrm{CH}_{3}\right), 2.12$ (s, 3H, $\left.\mathrm{CH}_{3}\right), 3.29$ (dd, 1H, J 5.4 and 12.9 Hz, H-1), 3.59 ( dd, J 12.9 and $4.3 \mathrm{~Hz}, 1 \mathrm{H}, \mathrm{H}-1$ '), 3.82-3.90 (m, 1H, H-2), 3.88 (dd, J 8.6 and $12.8 \mathrm{~Hz}, 1 \mathrm{H}, \mathrm{H}-6$ ), 4.04 (dd, $J 6.4$ and $8.6 \mathrm{~Hz}, 1 \mathrm{H}, \mathrm{H}-6), 4.13$ (dd, $J 2.1$ and 7.5 Hz,1H, H-3), 4.26 (q, J 6.4 Hz,1H, H-5), 5.02 (dd, J 2.1 and $6.4 \mathrm{~Hz}, 1 \mathrm{H}, \mathrm{H}-4) .{ }^{13} \mathrm{C} \mathrm{NMR}\left(\mathrm{CDCl}_{3}\right) \delta: 20.8,25.3,26.5$, 27.0, 51.1, 66.1, 70.4, 74.9, 75.7, 76.8, 109.5, 110.0, 170.3.

6-O-acetyl-1-azido-2,3-O-isopropylidene-D-glucitol (8a)

Method A. To a mixture containing acetyl azide 7 $(1.10 \mathrm{~g}, 3.36 \mathrm{mmol})$ and $\mathrm{MeOH}\left(5.0 \mathrm{~cm}^{3}\right)$, TsOH (catalyst, $0.1 \mathrm{mmol}$ ) was added. The mixture was stirred at $0{ }^{\circ} \mathrm{C}$ for $5 \mathrm{~h}$, neutralised with aqueous $\mathrm{NH}_{3}(10 \% \mathrm{v} / \mathrm{v})$ and evaporated. The residue was dissolved in $\mathrm{CH}_{2} \mathrm{Cl}_{2}$, washed with aqueous $\mathrm{NaCl}$ and dried over anhydrous $\mathrm{Na}_{2} \mathrm{SO}_{4}$. Filtration and evaporation of the solvent gave a residue that was purified on a chromatography column $(1: 1$ heptane-EtOAc) giving 8a $(0.33 \mathrm{~g}, 34 \%)$ and 7 (0.56 g, 51\%). ${ }^{1} \mathrm{H}$ NMR $\left(\mathrm{CDCl}_{3}\right) \delta: 1.45\left(\mathrm{~s}, 3 \mathrm{H}, \mathrm{CH}_{3}\right), 1.48$ (s, $\left.3 \mathrm{H}, \mathrm{CH}_{3}\right), 2.11\left(\mathrm{~s}, 3 \mathrm{H}, \mathrm{CH}_{3}\right), 2.64(\mathrm{~s}, 1 \mathrm{H}, \mathrm{OH}), 2.82(\mathrm{~d}, 1 \mathrm{H}, J$ $4.8 \mathrm{~Hz}, \mathrm{OH}), 3.35(\mathrm{dd}, 1 \mathrm{H}, J 5.0$ and $13.2 \mathrm{~Hz}, \mathrm{H}-1), 3.49$ (t, 1H, H-4), 3.57 (dd, 1H, J4.1 Hz, H-1'), 3.84 (br, s, 1H, H-5), 4.2-4.3 (m, 1H, H-2), 4.19 (dd, J 2.2 and 7.9 Hz, 1H, H-3), 4.25 (dd, J 5.5 and $12.3 \mathrm{~Hz}, 1 \mathrm{H}, \mathrm{H}-6), 4.43$ (dd, $J 2.7$ and $12.3 \mathrm{~Hz}, 1 \mathrm{H}, \mathrm{H}-6$ ) ); ${ }^{1} \mathrm{H} \mathrm{NMR}\left(\mathrm{CDCl}_{3} / \mathrm{D}_{2} \mathrm{O}\right) \delta: 1.45$ (s, 3H, $\left.\mathrm{CH}_{3}\right), 1.48\left(\mathrm{~s}, 3 \mathrm{H}, \mathrm{CH}_{3}\right), 2.11\left(\mathrm{~s}, 3 \mathrm{H}, \mathrm{CH}_{3}\right), 3.35$ (dd, J 5.0 and $13.2 \mathrm{~Hz}, 1 \mathrm{H}, \mathrm{H}-1), 3.49$ (dd, J 2.2 and $7.9 \mathrm{~Hz}, 1 \mathrm{H}, \mathrm{H}-4)$, 3.57 (dd, J 4.0 and 13.2 Hz, 1H, H-1'), 4.20-4.30 (m, 1H, H2), 4.19 (dd, J 2.2 and $7.9 \mathrm{~Hz}, 1 \mathrm{H}, \mathrm{H}-3), 4.25$ (dd, $J 5.5$ and $12.3 \mathrm{~Hz}, 1 \mathrm{H}, \mathrm{H}-6), 4.43$ (dd, 2.7 and12.3 Hz,1H, H-6'); ${ }^{13} \mathrm{C}$ $\operatorname{NMR}\left(\mathrm{CDCl}_{3}\right) \delta: 20.8,26.9,51.5,66.21,68.9,71.8,75.9$, 76.6, 110.1, 171.7 .

Method B. To a mixture containing 7 (0.53 g, $1.62 \mathrm{mmol})$ and $\mathrm{MeOH}\left(5.0 \mathrm{~cm}^{3}\right)$, 4-pyridinium toluenesulfonate $(0.05 \mathrm{~g})$ was added. The mixture was stirred at room temperature for $12 \mathrm{~h}$. Then, it was heated at $50{ }^{\circ} \mathrm{C}$. The reaction was stopped when TLC indicated the presence of a third compound. The solution was neutralised with aqueous $\mathrm{NH}_{3}(10 \% \mathrm{v} / \mathrm{v})$ then purified using a silica gel chromatographic column (1:1 heptane-EtOAc) giving 8a $(0.02 g, 4 \%), 8 b(0.13 g, 28 \%)$ and $7(0.20 g, 38 \%)$.

\section{4-O-acetyl-1-azido-2,3-isopropylidene-D-glucitol (8b)}

Colourless oil; ${ }^{1} \mathrm{H} \mathrm{NMR}\left(\mathrm{CDCl}_{3}\right): \delta 1.38\left(\mathrm{~s}, 3 \mathrm{H}, \mathrm{CH}_{3}\right)$, 1.42 (s, 3H, $\left.\mathrm{CH}_{3}\right), 2.10$ (s, 3H, $\left.\mathrm{CH}_{3}\right), 3.29$ (dd, $J 13.3$ and 5.0 Hz, 2H, H-1, H-6), 3.55 (dd, J 13.3 and 7Hz, 2H, H-1', 
H-6'), 3.63 (br, s, 2H, 2 OH$), 3.8-3.9$ (m, 2H, H-2 and H-5), 4.29 (dd, $J 8.2$ and $2.2 \mathrm{~Hz}, 1 \mathrm{H}, \mathrm{H}-3$ ), 4.88 (dd, $J 8.0$ and 2.3 $\mathrm{Hz}, 1 \mathrm{H}, \mathrm{H}-4) .{ }^{1} \mathrm{H} \mathrm{NMR}\left(\mathrm{CDCl}_{3} / \mathrm{D}_{2} \mathrm{O}\right) \delta: 1.42$ (s, 3H, $\left.\mathrm{CH}_{3}\right)$, 1.38 (s, 3H, $\left.\mathrm{CH}_{3}\right), 2.10$ (s, 3H, $\left.\mathrm{CH}_{3}\right), 3.34$ (dd, J 13.2 and $5.1 \mathrm{~Hz}, 1 \mathrm{H}, \mathrm{H}-1$ ), 3.53 (dd, $J 12.0$ and $5.1 \mathrm{~Hz}, 2 \mathrm{H}, \mathrm{H}-6$ '), $3.60\left(\mathrm{dd}, J 13.2\right.$ and $3.5 \mathrm{~Hz}, 1 \mathrm{H}, \mathrm{H}-1^{\prime}$ ), 3.69 (dd, $J 12.0$ and 3.2Hz, 1H, H-6'), 3.82-3.99 (m, 2H, H-2 and H-5), 4.33 (dd, $1 \mathrm{H}, J 8.3$ and $2.2 \mathrm{~Hz}, \mathrm{H}-3), 4.91(\mathrm{dd}, 1 \mathrm{H}, J 8.1$ and $2.1 \mathrm{~Hz}$, $\mathrm{H}-4) .{ }^{13} \mathrm{C} \mathrm{NMR}\left(\mathrm{CDCl}_{3}\right) \delta: 21.4,27.2,27.6,51.8,63.4$, 70.6, 71.2, 76.2, 77.0, 110.6, 171.7.

6-O-acetyl-1-azido-1-deoxy-2,3-isopropylidene-5-ptoluenesulfonyl-D-glucitol $(\mathbf{9 a})$ and 6-O-acetyl-1-azido1-deoxy-2,3-isopropylidene-5,6-di-p-toluenesulfonyl-Dglucitol $(\mathbf{9 b})$

To a mixture containing acetyl azide 8 (0.25 g, $0.87 \mathrm{mmol}$ ) and $5.0 \mathrm{~cm}^{3}$ of $\mathrm{Et}_{3} \mathrm{~N}$, under an inert atmosphere, cooled externally by an ice bath, $\mathrm{TsCl}(0.486 \mathrm{~g}, \mathrm{mmol})$ was added. The reaction was kept in the refrigerator for $12 \mathrm{~h}$. The filtrate was evaporated under reduced pressure to give crude 9a and 9b that were purified by a silica gel chromatographic column, (7:3 heptane-EtOAc) to yield 9a $(0.14 \mathrm{~g}, 36 \%)$ and 9b $(0.10 \mathrm{~g}, 19 \%)$.

9a. ${ }^{1} \mathrm{H}$ NMR $\left(\mathrm{CDCl}_{3}\right) \delta: 1.33\left(\mathrm{~s}, 3 \mathrm{H}, \mathrm{CH}_{3}\right), 1.39$ (s, 3H, $\left.\mathrm{CH}_{3}\right), 2.01$ (s, 3H, $\left.\mathrm{CH}_{3}\right), 2.45$ (s, 3H, $\left.\mathrm{CH}_{3}\right), 2.66$, (d, J9.8 Hz, $1 \mathrm{H},-\mathrm{OH}), 3.24$ (dd, $J 13.3$ and $4.4 \mathrm{~Hz}, 1 \mathrm{H}, \mathrm{H}-1), 3.54$ (dd, $J$ 13.3 and $4.0 \mathrm{~Hz}, 1 \mathrm{H}, \mathrm{H}-1^{\prime}$ ), 3.62 (t, J9.4, $\left.1.0 \mathrm{~Hz}, 1 \mathrm{H}, \mathrm{H}-4\right)$, 3.86 (dd, $1 \mathrm{H}, J 8.3$ and $1.1 \mathrm{~Hz}, \mathrm{H}-3), 4.10-4.16(\mathrm{~m}, 1 \mathrm{H}$, H-2), 4.32 (dd, $J 12.7$ and $2.5 \mathrm{~Hz}, 1 \mathrm{H}, \mathrm{H}-6), 4.50$ (dd, $J 12.7$ and 5.1 Hz, 1H, H-6'), 4.66-4.74 (m, 1H, H-5), 7.32 (d, J $8.1 \mathrm{~Hz}, 2 \mathrm{H},), 7.82(\mathrm{~d}, J 8.1 \mathrm{~Hz}, 2 \mathrm{H},) .{ }^{13} \mathrm{C} \mathrm{NMR}\left(\mathrm{CDCl}_{3}\right) \delta$ : 20.9, 21.7, 27.0, 26.8, 50.9, 62.5, 67.0, 75.6, 75.7, 79.0, $111.8,129.3,131.3,144.3,167.8$.

\section{4,5-anhydro-1-azido-1-deoxy-2,3-isopropylidene-D-} glucitol (10)

To a mixture containing the tosyl azide 9a $(0.10 \mathrm{~g}$, $0.22 \mathrm{mmol})$ and $5.0 \mathrm{~cm}^{3}$ of $\mathrm{MeOH}, \mathrm{K}_{2} \mathrm{CO}_{3}(0.114 \mathrm{~g}, 0.82$ mmol) was added. The solution was stirred at room temperature for $1 \mathrm{~h}$. The residue was purified by TLC, (1:1 heptane-EtOAc), giving compound $\mathbf{1 0}(0.029 \mathrm{~g}, 58 \%)$ as a colorless viscous oil. $v_{\max .} / \mathrm{cm}^{-1}: 3425 ; 2107,{ }^{1} \mathrm{H}$ NMR $\left(\mathrm{CDCl}_{3}\right) \delta: 1.42\left(\mathrm{~s}, 3 \mathrm{H}, \mathrm{CH}_{3}\right), 1.45\left(\mathrm{~s}, 3 \mathrm{H}, \mathrm{CH}_{3}\right), 2.00(\mathrm{br}, 1 \mathrm{H}$, $\mathrm{OH}), 3.15$ (dd, J 2.2 and $4.5 \mathrm{~Hz}, 1 \mathrm{H}, \mathrm{H}-4), 3.25$ (m, 1H, H5), 3.39 (dd, $J 4.5$ and $13.1 \mathrm{~Hz}, 1 \mathrm{H}, \mathrm{H}-1$ ), 3.59 (dd, $J 4.5$ and $13.1 \mathrm{~Hz}, 1 \mathrm{H}, \mathrm{H}-1$ '), 3.71 (br, d, $J 3$ and $13 \mathrm{~Hz}, 1 \mathrm{H}, \mathrm{H}-6), 3.92$ (dd, J 2.2 and $4.5 \mathrm{~Hz}, 1 \mathrm{H}, \mathrm{H}-3$ ), 3.96 (dd, J 2.4 and $13.1 \mathrm{~Hz}$, 1H, H-6'), 4.10-4.18 (m, 1H, H-2). ${ }^{13} \mathrm{C} \mathrm{NMR}\left(\mathrm{CDCl}_{3}\right) \delta$ : 26.6, 26.9, 51.5, 53.8, 55.6, 60.6, 77.5, 77.3, 110.6. 4,5-anhydro-1-azido-1-deoxy-2,3-isopropylidene-6-silylD-glucitol (11)

To a mixture containing $0.025 \mathrm{~g}(0.11 \mathrm{mmol})$ of epoxide $\mathbf{1 0}$ in $2.0 \mathrm{~cm}^{3}$ of DMF, tert-butyldimethylsilyl chloride $(0.032 \mathrm{~g}, 0.212 \mathrm{mmol})$ and imidazole $(0.02 \mathrm{~g}$, $0.34 \mathrm{mmol}$ ) were added under an inert atmosphere. The solution was stirred at room temperature for $24 \mathrm{~h}$. $\mathrm{CH}_{2} \mathrm{Cl}_{2}$ was added to the residue, and it was washed with an aqueous solution of $\mathrm{NaCl}$ and dried over anhydrous $\mathrm{Na}_{2} \mathrm{SO}_{4}$. Then, the solvent was removed under reduced pressure. The residue was purified by PLC (6:4 heptaneEtOAc), giving 11 (0.02 mg, 53\%) and 10 (0.010 g, 42\%). ${ }^{1} \mathrm{H} \mathrm{NMR}\left(\mathrm{CDCl}_{3}\right) \delta: 0.06\left(\mathrm{~s}, 3 \mathrm{H}, \mathrm{CH}_{3}\right), 0.07$ (s, 3H, $\left.\mathrm{CH}_{3}\right)$, 0.89 (s, 9H, $\mathrm{CH}_{3}$ ), 1.42 (s, 3H, $\left.\mathrm{CH}_{3}\right), 1.45$ (s, 3H, $\left.\mathrm{CH}_{3}\right), 3.00$ (dd, J 2.2 and $4.7 \mathrm{~Hz}, 1 \mathrm{H}, \mathrm{H}-4), 3.09-3.14$ (m, 1H, H-5), 3.35 (dd, $J 4.6$ and $13.2 \mathrm{~Hz}, 1 \mathrm{H}$ ), 3.58 (dd, $J 4.2$ and 13.2 $\mathrm{Hz}, 1 \mathrm{H}, \mathrm{H}-1$ ), 3.75 (dd, J 4.0 and $12.1 \mathrm{~Hz}, 1 \mathrm{H}, \mathrm{H}-6$ '), 3.58 (dd, $J 4.2$ and $13.2 \mathrm{~Hz}, 1 \mathrm{H}, \mathrm{H}-1^{\prime}$ ), 3.83 (dd, $J 3.4$ and 12.1 $\mathrm{Hz}, 1 \mathrm{H}, \mathrm{H}-6), 3.88$ (dd, 1H, J 4.7 and $8.1 \mathrm{~Hz}, \mathrm{H}-3$ ), 4.10-4.16 (m, $1 \mathrm{H}, \mathrm{H}-2) .{ }^{13} \mathrm{C} \mathrm{NMR}\left(\mathrm{CDCl}_{3}\right) \delta:-5.4,18.3$, $25.8,26.6,26.8,51.5,54.2,55.7,62.3,76.6,77.3,110.5$

\section{1,5-dideoxy-1,5-imino-2,3-O-isopropylidene-D-glucitol} (13)

To a solution of $0.02 \mathrm{~g}$ of epoxide $11(0.057 \mathrm{mmol})$ in $5.0 \mathrm{~cm}^{3}$ of $\mathrm{EtOH}, \mathrm{NaBH}_{4}$ was added $(0.002 \mathrm{~g})$. The reaction was stirred at room temperature for $12 \mathrm{~h}$. As no reaction was observed, the mixture was heated to $40{ }^{\circ} \mathrm{C}$. After $24 \mathrm{~h}$ of reaction, more $\mathrm{NaBH}_{4}(0.003 \mathrm{~g})$ was added and the solution was stirred at $40{ }^{\circ} \mathrm{C}$ for next $12 \mathrm{~h}$. $\mathrm{CH}_{2} \mathrm{Cl}_{2}$ was added to the mixture, which was then washed with aqueous $\mathrm{NaCl}$, dried over anhydrous $\mathrm{Na}_{2} \mathrm{SO}_{4}$ and the solvent was removed under reduced pressure.

The residue was purified by TLC (1:1 heptane-EtOAc), affording $13(0.008 \mathrm{~g}, 48 \%)$. $[\alpha]_{\mathrm{D}}{ }^{25}=+23.6\left(\mathrm{CHCl}_{3}\right)$; (lit. ${ }^{12}$ $\left.+20.1\left(\mathrm{CHCl}_{3}\right)\right)$; ${ }^{1} \mathrm{H} \mathrm{NMR}\left(\mathrm{CDCl}_{3}\right) \delta 0.08\left(\mathrm{~s}, 3 \mathrm{H}, \underline{\mathrm{CH}}_{3}-\mathrm{Si}\right)$, 0.10 (s, 3H, $\underline{\mathrm{CH}}_{3}-\mathrm{Si}$ ), 0.91 (s, 9H, $\mathrm{CH}_{3}$ (t-butyl-Si-), 1.47 (s, $6 \mathrm{H}, 2 \mathrm{CH}_{3}$ ), 2.48-2.58 (m, 1H, H-5), 2.75 (dd, $J 11.2$ and $\left.12.7 \mathrm{~Hz}, 1 \mathrm{H}, \mathrm{H}-1_{\mathrm{ax}}\right), 3.28-3.36$ (m, 2H, H-1' and H-2), 3.42 (t, J 8.7 Hz, 1H, H-3), 3.72 (t, J 8.7 Hz, 1H, H-4), 3.79 (dd, $J 4.7$ and $9.8 \mathrm{~Hz}, 1 \mathrm{H}, \mathrm{H}-6), 3.91$ (dd, $J 4.2$ and $9.8 \mathrm{~Hz}, 1 \mathrm{H}$, $\mathrm{H}-6$ ' $) .{ }^{13} \mathrm{C} \mathrm{NMR}\left(\mathrm{CDCl}_{3}\right) \delta:-5.5,18.2,25.9,26.3,46.7,60.8$,

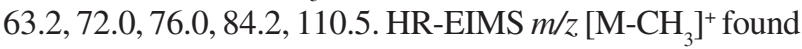
302.167. Calc. for $\mathrm{C}_{14} \mathrm{H}_{28} \mathrm{NO}_{4} \mathrm{Si}: 302.179$.

\section{Acknowledgments}

The authors thank FAPESP, FAEP-UNICAMP and CAPES for financial support. Thanks also are due to Dr. 
Carol Collins (Instituto de Quimica, UNICAMP) for linguistic and grammatical corrections.

\section{References}

1. MacNaught, A.D.; Carbohydr. Res. 1997, 297, 1.

2. Ichikawa, Y.; Igarashi, Y.; Ichikawa, M.; Suhara, Y.; J. Am. Chem. Soc. 1998, 120, 3007.

3. Look, G.C.; Fotsch, C.H.; Wong, C-H.; Acc. Chem. Res. 1993, 26, 182.

4. Merrer, Y.L.; Poitout, L.; Depezay, J-C.; Dosbaa, I.; Geoffroy, S.; Foglietti, M-J.; Bioorg. Med. Chem. 1997, 5, 510.

5. Xu, Y-M.; Zhou, W-S.;Tetrahedron Lett. 1996, 37,1461.

6. Kilonda, A.; Compernolle, F.; Toppet, S.; Hoornaert, G.J.; J. Chem. Soc. Chem. Commun. 1994, 2147.

7. Hughes, B.; Rudge, J.; Nat. Prod. Rep. 1994,135.

8. Kilonda, A.; Compernolle, F.; Peeters, K.; Joly, G. J.; Toppet, S.; Hoornaert, G.J.; Tetrahedron 2000, 56, 1005.

9. Paulsen, H.; Sangster, I; Heynes, K.; Chem. Ber. 1967, 100, 802.

10. Kinast, G.; Schedel, M.; Angew Chem. Int. Ed. Eng. 1981, 20, 805.

11. Behling, J.; Medich, J.R.; Scaros, M.G.; Prunier, M.; Weier, R. M.; Khanna, I.; Synth. Commun 1991,21, 1383.

12. Setoi, H.; Takeno, H.; Hashimoto, M.; Chem. Pharm. Bull 1986, 34, 2642.
13. Setoi, H.; Takeno, H.; Hashimoto, M.; Tetrahedron Lett. 1985 , $26,4617$.

14. Doane, W.M.; Shasha, B.S.; Russel,C.R.; Rist, C.E.; J. Org. Chem. 1967, 32, 1080.

15. Matos, C. R. R; Lopes, R. S. C.; Lopes,C. C.; Synthesis 1999, $4,571$.

16. Somfai, P.; Marchand, P.; Torsell, S.; Lindström, U. M.; Tetrahedron 2003, 59, 1293.

17. Han, H.; Tetrahedron Lett. 2003,44,1567.

18. Comins, D.L.; Fulp, A.B.; Tetrahedron Lett. 2001,42,6839.

19. Kato, L.; M. Sc Dissertation, Universidade Estadual de Campinas, Brazil, 1996.

20. Silverstein,R.M.; Bassler, G.C.; Morril, T.C.; Spectrometric Identification of Organic Compounds, $5^{\text {th }}$ ed., Wiley: New York, 1991

21. Kato, L.; Braga, R. M.; Mag. Res. Chem. 1999, 37, 447.

22. Reactivos de Coloración para Cromatografía en Capa Fina y en Papel, E. Merck, Darmstadt: Germany, 1972, p. 92.

23. Perrin, D. D.; Armarego, L.F.; Purification of Laboratory Chemicals, $3^{\text {rd }}$ ed., Pergamon Press:Oxford, 1988

24. Kuzsmann, J.; Sohar, P.; Carbohydr. Res. 1979, 74, 187.

25. Kuzsmann, J.; Kiss, L.; Carbohydr. Res. 1986, 153, 45.

Received: March 31, 2003

Published on the web: September 1, 2003

FAPESP helped in meeting the publication costs of this article. 